\title{
Advancing Structure-Based Drug Design: IMCA-CAT Responds to Emerging Needs of the Pharmaceutical Industry
}

\author{
J Yoder ${ }^{1}$, A Mulichak ${ }^{2}$, J Muir ${ }^{2}$, E Zoellner ${ }^{2}$, J Digilio ${ }^{2}$, E Duguid ${ }^{2}$, L Keefe ${ }^{3}$ \\ ${ }^{1}$ IMCA-CAT, Lemont, IL, ${ }^{2}$ IMCA-CAT / HWI @ Advanced Photon Source, Argonne National Lab, \\ Lemont, IL, ${ }^{3}$ IMCA-CAT / HWI @ Advanced Photon Source, Argonne National Lab \\ jyoder@imca-cat.org
}

For over 20 years, the Industrial Macromolecular Crystallography Association Collaborative Access Team (IMCACAT) has focused exclusively on accelerating drug discovery and development by delivering synchrotron-based diffraction data. Rapid turnaround of high quality and low-cost structural data is essential in ensuring success in structure-based drug design and an increasing number of computer-aided drug design projects. Today, IMCA-CAT operates a state-of-the-art MX beamline; operating continuously with fully unattended data collection for overnight means that $>90 \%$ of samples are turned around within one day. IMCA-CAT can handle crystals in a variety of pins and pucks, the data are automatically processed and then rapidly delivered to researchers in a secure, encrypted, and reliable software framework. In addition to MX data collection, IMCA-CAT serves as the conduit for SAXS data collection and structure-based consultancy services. On the horizon are modes of access to Hauptman-Woodward Medical Research Institute's centers for high-throughput crystallization screening, cryo-EM structure determination, and microcrystal electron diffraction. IMCA-CAT is the one-stop shop for all the services that the next generation pharmaceutical structural biologist will need. Opportunities abound for researchers in all pharmaceutical and biotechnology organizations to access IMCA-CAT for structural services.

Acta Cryst. (2020). A76, a211 